\title{
Electron transport in coupled chains of interacting fermions with impurities
}

\author{
E. Arrigoni, B. Brendel and W. Hanke \\ Institut für Theoretische Physik, Universität Würzburg, D-97074 Würzburg, Germany
}

\begin{abstract}
We study the low-temperature transport of a doped two-chain ladder system of interacting fermions in the presence of a barrier or of a low concentration of impurities. Above a certain value of the interaction, the conductance is suppressed, like for a single chain, despite the presence of dominant superconducting correlations. There is, however, a region of repulsive interaction where perfect transmission across the barrier occurs unlike the single-chain case. We provide a possible explanation for the temperature maximum of the resistivity in the normal state of $\mathrm{Sr}_{0.4} \mathrm{Ca}_{13.6} \mathrm{Cu}_{24} \mathrm{O}_{42-\delta}$.
\end{abstract}

PACS numbers : 71.10.Pm, 72.10.Fk, 71.10.Hf

Transport properties in one-dimensional (1D) interacting fermion systems, i.e. Luttinger liquids [1], are remarkably affected by the interaction. Indeed, it has been shown [2] that the linear conductance across an impurity is completely suppressed in the zero-temperature limit for repulsive interaction no matter how weak the barrier potential. Recently, the question of what happens when a small number of Luttinger Liquids are coupled by a single-particle hopping has received particular interest 3 6]. Coupled chains represent the first step towards a two-dimensional system, but the important effects of one-dimensionality in the single chains can still be treated in an accurate way. Various numerical calculations and renormalization-group (RG) treatments agree about the fact that the two-chain system is in a spin-gapped state with dominant "odd-mode" (the analogy of d-wave in two chains) superconducting correlations [3 6]. The apparent similarity with the high- $\mathrm{T}_{c}$ superconductors has suggested the idea that the pairing mechanism in the high- $\mathrm{T}_{c}$ materials could be of a similar origin. This theoretical interest is supplemented by experimental realizations of systems of 2 to 5 coupled chains, such as $\mathrm{VO}_{2} \mathrm{P}_{2} \mathrm{O}_{7}, \mathrm{Sr}_{n-1} \mathrm{Cu}_{n+1} \mathrm{O}_{2 n}$, and others [7]. In addition to the observation of a remnant spin gap in the doped metallic state, superconductivity has been recently observed at high pressure in the ladder material $\mathrm{Sr}_{0.4} \mathrm{Ca}_{13.6} \mathrm{Cu}_{24} \mathrm{O}_{42-\delta}$ [8]. Finally, narrow GaAs quantum wires with few transverse channels can also be represented by coupled-chain systems in the intermediate situation between one and many channels [9].

In this Letter, we study how the results of Refs. [2] for the transport of interacting electrons across an impurity in a 1D Luttinger liquid evolve when two $1 \mathrm{D}$ systems are coupled together. In particular, we analyze the influence of the gaps (in the spin and in the "odd" charge modes) and of the superconducting correlations, present in the two-chain system, on transport properties. Specifically, we study the conductance of a system of two Hubbard-like chains coupled by a single-particle hopping in the presence of a barrier or a low impurity concentration. This system is a good description for a two-channel conductor and for the ladder materials. Similarly to the pure 1D case [2], we obtain that the conductance across the impurity is completely suppressed when- ever the "even charge" correlation exponent $K_{\rho,+}$ is less than unity 10. However, there is an additional region of repulsive electron-electron interaction, $U \lesssim U_{c}$, where $K_{\rho,+}>1$ and perfect transmission across the impurity occurs. A similar result is obtained for the three-chain case where, in the pure case, more gapless modes are present [11,12]. If we limit ourselves to weak impurities and temperature $T$ smaller than all the gaps, then our results for the $T$-dependence of the conductivity $\sigma$ agree with a recent treatment of a large concentration of weak impurities [13].

We consider a Hamiltonian of the general form

$$
H=H_{\text {chain }, t}+H_{\text {chain }, U}+H_{\perp}+V_{b},
$$

where $H_{\text {chain }, t}+H_{\text {chain }, U}$ describes $n_{c}(=2)$ uncoupled 1D Hubbard chains, with $H_{\text {chain, } t}$ denoting the intrachain hopping $t$, and $H_{\text {chain }, U}$ the on-site repulsion $U$. The chains are coupled by an inter-chain hopping term $H_{\perp}$ with hopping amplitude $t_{\perp}$ (with open boundary conditions in the perpendicular direction). $V_{b}$ is the impurity potential described in detail below. It should be pointed out that the qualitative results do not depend significantly on the detailed structure of the model. One can easily extend the method and the results to other systems with, e. g., a more general band structure than the simple cosine band and a nearest-neighbor interchain and/or intrachain interaction. In the weak-coupling case, one first diagonalizes the single-particle part of the Hamiltonian $\left(H_{\text {chain }, t}+H_{\perp}\right)$, obtaining $n_{c}$ bands. The interaction $H_{\text {chain }, U}$ written in the band representation includes different classes of processes that scatter particles or holes at the "Fermi surface" (which consists of $2 n_{c}$ points). These interactions have to be treated by a RG analysis, since perturbation theory is divergent. The clean case $\left(V_{b}=0\right)$ has been considered by several authors (Refs. [5,6] for two chains and [11] for three chains).

One introduces four boson fields $\varphi^{(s, q)}$ and four dual fields $\theta^{(s, q)}$ for the two spins $s= \pm 1$ and for the two bands $q=0, \pi$. Taking the symmetric and antisymmetric combinations of the band and of the spin part, one obtains the fields $\varphi_{\rho, \pm}, \varphi_{\sigma, \pm}, \theta_{\rho, \pm}$, and $\theta_{\sigma, \pm}$ [10]. The RG calculations of Refs. [5]6] yield a mass gap with short-range correlations for both "spin" degrees of freedom $\varphi_{\sigma, \pm}$ and for the "odd-charge" degree of freedom 
$\theta_{\rho,-}$ [14]. In this phase, "odd mode" pairing correlations decay rather slowly (as $r^{-1 /\left(2 K_{\rho,+}\right)}$ ) and dominate with respect to other correlations in the weak-coupling limit.

Next, we introduce the perturbation due to an impurity in a single chain, or due to a symmetric "barrier" in both chains:

$$
V_{b}=\int d x \sum_{q_{1}, q_{2}, s} V_{q_{1}, q_{2}}(x) \psi_{s, q_{1}}^{\dagger}(x) \psi_{s, q_{2}}(x)
$$

where $\psi_{s, q}(x)$ destroys a fermion with spin $s$ on band $q$ at site $x$. Here, the impurity potentials $V_{q_{1}, q_{2}}(x)$ satisfy $V_{0,0}(x)=V_{\pi, \pi}(x)$ and, for a symmetric barrier, $V_{0, \pi}(x)=$ 0 . Introducing the boson representation and considering a potential that is restricted to a small region around $x=$ 0 , one obtains three contributions for the perturbation : a backscattering intraband term, i.e.

$V_{\text {intra }} \sim \sum_{s} v_{0,0}\left(2 k_{F}\right) \cos \left(\varphi_{\rho,+}+s \varphi_{\sigma,+}\right) \cos \left(\varphi_{\rho,-}+s \varphi_{\sigma,-}\right)$

and two interband (back- and forward-scattering) terms (the forward-scattering intraband term can, as usual, be absorbed by a canonical transformation). The interband terms have a form similar to $V_{\text {intra }}$, except that some cosines are replaced with sines and the phase fields $\varphi_{\nu,-}$ (for the backscattering) or $\varphi_{\nu,+}$ (for the forward scattering) are replaced with $\theta_{\nu,-}(\nu=\rho$ or $\sigma)$. In Eq. (3), $v_{0,0}(k)$ is proportional to the Fourier transform of $V_{0,0}(x)$ with longitudinal momentum $k$ [we have approximated $v_{0,0}\left(2 k_{F}\right)$ by $\left.v_{q, q}\left(2 k_{F}(q)\right)\right]$, and the fields are implicitly considered to be at the origin, $x=0$.

As pointed out in Ref. [2], the perturbation will evolve under RG into a more general form consistent with the symmetries of the Hamiltonian. In our case, $V_{b}$ is symmetric under rotation by $2 \pi$ of any of the fields $\varphi_{\nu,+}$, $\varphi_{\nu,-}$, or $\theta_{\nu,-}$. Moreover, a simultaneous rotation of $\pi / 2$ of all six of these fields or a simultaneous rotations by $\pi$ of some groups of two or three fields (such as, e. g., $\varphi_{\rho,+}$, $\varphi_{\rho,-}$, and $\theta_{\sigma,-}$ ) leaves the perturbation (and also the rest of the Hamiltonian) invariant. The general perturbation operator which is consistent with these symmetries develops under the RG and can be cast into the form

$V_{b}^{\prime}=\sum_{n_{\nu, \pm}^{\varphi}, n_{\nu,-}^{\theta}} \mathcal{V}(\underline{n}) \exp \left[i \sum_{\nu=(\rho, \sigma)}\left(n_{\nu,-}^{\theta} \theta_{\nu,-}+\sum_{p= \pm} n_{\nu, p}^{\varphi} \varphi_{\nu, p}\right)\right]$

Here, the "Fourier coefficients" $\mathcal{V}(\underline{n})$ are nonzero only for values of the integers $\underline{n} \equiv\left\{n_{\nu, \pm}^{\{\varphi, \theta\}}\right\}$ satisfying certain constraints imposed by symmetries. A calculation, in principle similar to ours, has been carried out by T. Kimura and coworkers for the two-chain case 115 . However, these authors restrict their analysis to the bare perturbations [Eq. (3) plus the interband terms], which are completely suppressed due to the presence of the gap in the $\theta_{\rho,-}$ and $\varphi_{\sigma,-}$ field (at least in phases I and III of Ref. [15]). Thus, they argue that the system should behave as a perfect conductor even in the presence of impurities. We show here, however, that their result is invalid if one carefully takes into account the additional terms in Eq. (4) generated under the RG. Indeed, perturbation terms where the subset of integers $n_{\rho,-}^{\varphi}$ and $n_{\sigma,-}^{\theta}$ vanishes survive. The most relevant terms are the ones with the smallest $n_{\rho,+}^{\varphi}$ consistent with the constraints on the $n_{\nu, \pm}^{\{\varphi, \theta\}}$ discussed above. One (nontrivial) solution is given, for example, by the perturbation term $v_{2} \cos \left(2 \varphi_{\rho,+}\right)$. Here, we have exploited inversion symmetry and replaced the gapped fields with their average (vanishing) values. To see how such a term can originate, consider the intraband part of the initial perturbation in Eq. (3). Although its coefficient $v_{0}(E)$ (whose bare value is $v_{0}\left(E_{F}\right) \equiv v_{0,0}\left(2 k_{F}\right)$ ) is suppressed during the RG transformation [15], $v_{2}$ has the RG equation

$$
\frac{d v_{2}(E)}{-d \log E}=-\frac{1}{8}\left[v_{0}(E)\right]^{2}+\left(1-K_{\rho,+}\right) v_{2}(E),
$$

where $E$ is the characteristic energy parameterizing the RG flow. Therefore $v_{2}$, although having vanishing initial conditions $v_{2}\left(E=E_{F}\right)=0$, is obtained under $\mathrm{RG}$ from the irrelevant term $v_{0}(E)$ and is itself relevant whenever $K_{\rho,+}<1$. Physically, the fermionic representation of the operator associated with $v_{2}$ is

$\psi_{+, s, 0}^{\dagger}(x) \psi_{+, s, \pi}^{\dagger}(x) \psi_{-, s, \pi}(x) \psi_{-, s, 0}(x)$ [10], which represents an interband electron-pair backscattering operator. This term is absent in the bare impurity potential, but is obtained to second-order perturbation theory in $V_{\text {intra }}$.

When $K_{\rho,+}>1$, the perturbation $v_{2}$ becomes irrelevant and the low-temperature ( $T$ smaller than all the gaps) conductance $G(T)$ behaves as $\delta G(T) \equiv G(T)-$ $G_{0} \propto-v_{2}(T)^{2} \propto-v_{0}^{4} T^{2\left(K_{\rho,+}-1\right)}$ [16] where $G_{0}$ is the value of the conductance without impurities, $v_{0} \equiv$ $v_{0}\left(E_{F}\right)$, and $v_{2}(T)$ is the solution of Eq. (5). For $K_{\rho,+}<1$, the expression for $\delta G(T)$ is valid only down to a temperature $T=T^{*}$ with $\delta G\left(T^{*}\right) / G_{0} \lesssim 1$. For a low impurity concentration $n$, such that the temperature is larger than the "discretization energy" $T_{d i s} \equiv v_{F} n$, the impurities can effectively be treated independently and their contribution to the total resistance adds up incoherently [2]. Thus, the resistivity $\rho$ behaves like $\rho \cdot \propto v_{0}^{4} n T^{2\left(K_{\rho,+}^{-1)}\right.}\left(\right.$ down to $\left.T=\max \left(T^{*}, T_{d i s}\right)\right)$, in Jagreement with Ref. [13].

When $K_{\rho,+}<1$ and $T<T^{*}$, the renormalized perturbation diverges and one has to treat the case of large $v_{2}$. In this limit, and for a single impurity, we can fermionize back the operator associated with $v_{2}$ and consider two decoupled semi-infinite (spinless) Luttinger Liquids connected via a weak tunneling matrix element $t_{2}$ [2]. This gives a perturbation dual to $v_{2}: t_{2} \cos \left(2 \theta_{\rho,+}(0)\right)$. Similarly to [2], one obtains a conductance $G(T) \propto$ $t_{2}^{2} T^{\left(2 / K_{\rho,+}\right)-2}$, which vanishes at low temperatures. 
Therefore, the transmission through an impurity in a two-chain system is similar to the case of a single chain. For $K_{\rho,+}<1$ the impurity is perfectly insulating, whereas perfect transmission occurs in the case of $K_{\rho,+}>1$, independent of the barrier's strength and symmetry. Physically, this can be understood by considering that $v_{2}$ is associated with a charge-density wave (CDW) operator with wave vector $2 k_{F}(0)+2 k_{F}(\pi) \equiv 4 k_{F}$ whose correlations decay like $r^{-2 K_{\rho,+}}$. The argument of Ref. [2] can thus be applied to these $4 k_{F}$ CDW correlations (since the "regular" $2 k_{F}$ ones are suppressed [15]). When $K_{\rho,+}<1$ the $4 k_{F}$ CDW correlations are long-range enough and are pinned by an arbitrarily weak impurity at low energies.

Nevertheless, there is an essential difference between the single- and the two-chain system, since $K_{\rho,+}$ is renormalized by an amount $\delta K_{\rho,+} \propto\left(\delta v_{F} / v_{F}\right)^{2}$ by the presence of the gap $\Delta_{o}$ in the "odd" modes [6] (here, $\delta v_{F}$ is the difference between the Fermi velocities $v_{F}$ of the bonding and antibonding bands). This renormalization can be considered as an effective attraction mediated by the odd gapped modes. The RG equation for $K_{\rho,+}$ as a function of the flux parameter $\Gamma$ ( $\Gamma$ is proportional to $l=-\log E / E_{F}$ in our notation) is reported in Ref. [6] (in order to match our notation, one has to replace $K_{\rho,+} \rightarrow 1 / K_{\rho,+}$ in that reference). The exact value of $\delta K_{\rho,+}$ cannot be determined even in the $U / t \rightarrow 0$ limit, since it depends on the point $l^{*}$ where the RG flow is stopped. A reasonable lower bound and estimate for $\delta K_{\rho,+}$ and for the critical value $U_{c}$, which separates regions $K_{\rho,+}<1$ and $K_{\rho,+}>1$ in Fig. 1 can be obtained in the following way: Notice that $\delta K_{\rho,+}$ contains two contributions: (i) an $l$-independent contribution obtained by neglecting the RG flow of the couplings (i. e. by taking $l=0$ and by integrating out the gapped modes), and (ii) an additional contribution due to the renormalization of the couplings which depends on $l$. One can show that (i) sets a lower bound for $\delta K_{\rho,+}$ and that the contribution of (ii) is much smaller than (i) and only weakly $l$-dependent.

In Fig. 1, we plot $U=U_{c}$ as a function of doping $\delta$ (away from half-filling) for the isotropic case $t_{\perp}=1$, by taking into account in the dashed line only the contribution (i) and by including in the full line additionally the contribution (ii) 17]. For large doping there is a sizable region of repulsive interaction for which the transmission across the impurity is perfect. This effect will be particularly relevant for systems in which the Hubbard repulsion $U \lesssim t$, as for example in carbon nanotubes [18] and in GaAs quantum wires. Exact numerical results for small $U$ 19 yield the opposite effect, namely $K_{\rho,+}$ seems to be reduced with respect to the single-chain value. We believe, however, that in these numerical studies the value of $K_{\rho,+}$ is underestimated since the system size considered (6 lattice spacings $a_{0}$ ) is smaller than the spin correlation length $\xi_{s} \propto v_{F} / \Delta_{s}$, which gives $\xi_{s} \gtrsim 30-40 a_{0}$ [4], where $\Delta_{s}$ is the spin gap.

For a finite impurity concentration, the resistivity $\rho(T)$

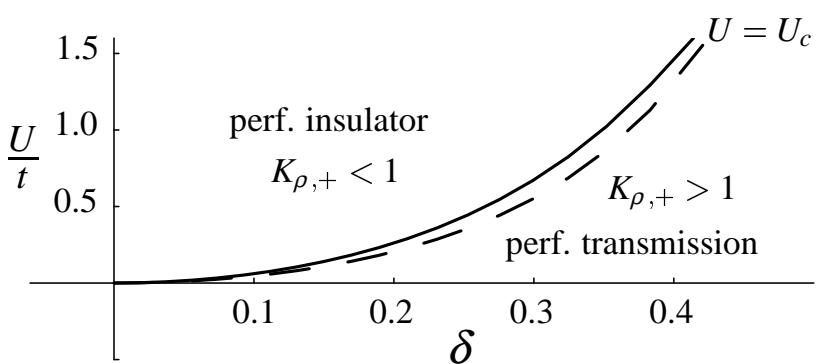

FIG. 1. Phase diagram for the two-chain system with a barrier or with a low impurity concentration. The two estimates for the boundary $U=U_{c}$ are obtained according to contribution (i) only (dashed) and additionally (ii) (full) as explained in the text.

diverges at low temperatures in the region in which $K_{\rho,+}<1$, whereas it decreases in the region in which $K_{\rho,+}>1$ [20]. At temperatures larger than the gap, $T \gg \Delta_{o} \approx \Delta_{s}$ 21], the resistivity is dominated by the scattering with the $2 k_{F}$ potential $v_{0}$. Its behavior [22] is $\rho(T) \propto v_{0}^{2} n T^{\left(K_{\rho}-1\right) / 2}$, yielding an increasing resistivity with decreasing $T$ (for $U>0$ ).

For a more quantitative evaluation of $\rho(T)$ and in order to find the behavior of $\rho(T)$ in the crossover regime for $T$ of the order of $\Delta_{o}$, we use the perturbative expression $\rho(T) \propto c_{0} v_{0}(T)^{2}+c_{2} v_{2}(T)^{2}$, where $c_{0}$ and $c_{2}$ are constants [23]. For these temperatures, $v_{0}(T)$ can be described by an activated behavior $v_{0}(T) \approx$ $\left(T / E_{F}\right)^{\left(K_{\rho}-1\right) / 4} v_{0} \exp \left(\Delta_{o} / E_{F}-\Delta_{o} / T\right)$, whereas $v_{2}$ can be evaluated from Eq. (5) [12]. In the weak-impurity regime which we are considering, the parameter $\tilde{v} \equiv$ $v_{0} \sqrt{c_{2} / c_{0}}$ [23] takes a value $\tilde{v} \lesssim 1$. In this case $\rho(T)$ is dominated by the $2 k_{F}$-scattering potential $v_{0}$ from higher temperatures down to a crossover temperature $T=T_{m} \lesssim \Delta_{o}$, with $T_{m} \sim \Delta_{o} /(2 \log (2 / \tilde{v}))$. Therefore, $\rho(T)$ eventually decreases down to this crossover temperature $T_{m}$. For $T<T_{m}$ the $4 k_{F}$-scattering $v_{2}$ becomes dominant and, if $K_{\rho,+}<1, \rho(T)$ dramatically increases eventually diverging for low $T$.

In Fig. 2, we plot the behavior of $\rho(T)$ for different values of the parameters $\tilde{v}$ and $\Delta_{s} / \Delta_{o}$, and for $K_{\rho} \approx K_{\rho,+}=0.6$, which corresponds to $U / t \approx 8.0$ 24] and doping $\delta \sim 0.25$ [20,25]. The full line is obtained by taking a large ratio $\Delta_{s} / \Delta_{o} \approx 4$ between the spin and the odd-mode gaps. For this large ratio and in the regime $2 \Delta_{o} \ll T \lesssim 2 \Delta_{s}, \rho(T)$ is dominated by the factor $\propto T^{\left(K_{\rho}-3\right) / 2}$, since in this regime the fields $\varphi_{\sigma, \pm}$ have a vanishing expectation value [26]. This induces the down turn of $\rho(T)$ at these higher temperatures.

The results shown by the full line in Fig. 2 may provide one possible explanation of the experimentally observed maximum of $\rho(T)$ at $T \approx 100 \mathrm{~K}$ in the normal state of $\mathrm{Sr}_{0.4} \mathrm{Ca}_{13.6} \mathrm{Cu}_{24} \mathrm{O}_{42-\delta}$ [8,24]. As mentioned above the large ratio $\Delta_{s} / \Delta_{o}$ guarantees the experimentally observed down turn of $\rho(T)$ above its maximum. Furthermore, our choice of $\Delta_{s} / \Delta_{o} \approx 4$ is consistent with the following estimate: from the full line in Fig. 
$2, \Delta_{s}$ has a value of about 1.3 times the temperature for which $\rho(T)$ has a maximum. Comparing with experiment, this fixes $\Delta_{s}$, i.e. $\Delta_{s} \approx 130 \mathrm{~K}$. On the other hand, if we take the value for the undoped material, $\Delta_{s}(n=1) \approx 430 \mathrm{~K}[\mathbb{8}]$, and estimate the doping-induced reduction (according to the second of Ref. 四), we obtain $\Delta_{s}(n=0.75) \approx 0.33 \Delta_{s}(n=1) \approx 140 \mathrm{~K}$. This is consistent with our previous result. The increase of $\rho(T)$ at $T \gtrsim 200 \mathrm{~K}$ in the experiments is probably due to contributions from electron-electron and electron-phonon scatterings. Notice that an appropriate description of the superconducting transition cannot be obtained by our simplified effectively one-dimensional model. We finally comment on the pressure dependence of $\rho(T)$ shown by the experiments. Application of hydrostatic pressure increases the carrier density in the ladders [27], thus decreasing the overall resistivity (as observed experimentally), and at the same time slightly reducing the values of the gaps and, consequently, the peak position in $\rho(T)$.

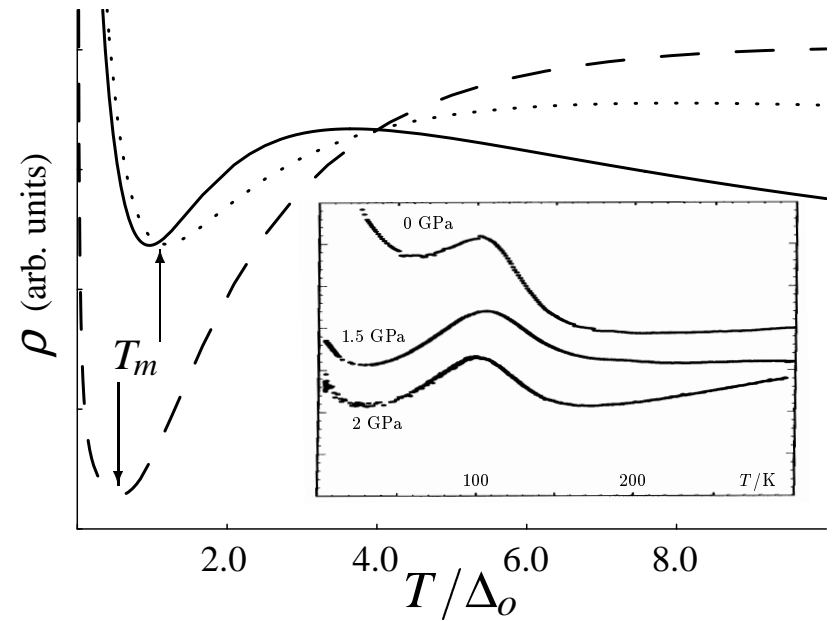

FIG. 2. Behavior of the resistivity $\rho$ as a function of temperature (in units of the odd-mode gap $\Delta_{o}$ ) for values of the parameters (as explained in the text): $\tilde{v}=0.4$ and $\Delta_{s} / \Delta_{o}=4$. (full line); $\tilde{v}=0.4$ and $\Delta_{s}=\Delta_{o}$ (dotted); $\tilde{v}=0.1$ and $\Delta_{s}=\Delta_{o}$ (dashed). For all curves, $K_{\rho}=K_{\rho,+}=0.6$ and $E_{F} / \Delta_{o}=150$. Inset: $\rho(T)$ for the normal state of $\mathrm{Sr}_{14-x} \mathrm{Ca}_{x} \mathrm{Cu}_{24} \mathrm{O}_{42-\delta}$ from Ref. [8] for different values of pressure. (With kind permission of the authors).

In conclusion, we have studied the transport properties of two-chain ladder systems in the presence of a barrier or of a low impurity concentration. We find a small region of repulsive interaction $0<U<U_{c}$ [cf. Fig. 1], for which the system is a perfect conductor for $T_{d i s} \lesssim T \lesssim \Delta_{o}$. We also suggested a possible explanation for the normal state behavior of the resistivity in $\mathrm{Sr}_{14-x} \mathrm{Ca}_{x} \mathrm{Cu}_{24} \mathrm{O}_{42-\delta}$.

We thank J. Voit, E. Orignac, H. J. Schulz, and R. M. Noack for stimulating discussions. This work was partially supported by the EC network program ERBCHRX-CT940438, by the Bavarian high-Tc program FORSUPRA and by the BMBF (05 605 WWA 6). E. A. acknowledges research support from the EC-TMR program ERBFMBICT950048.
[1] F. D. M. Haldane, J. Phys. C 14, 2585 (1981).

[2] C. L. Kane and M. P. A. Fisher, Phys. Rev. B 46, 15233 (1992); A. Furusaki and N. Nagaosa, Phys. Rev. B 47, 4631 (1993).

[3] T. M. Rice, S. Gopalan, and M. Sigrist, Europhys. Lett. 23, 445 (1993).

[4] R. M. Noack, S. R. White, and D. J. Scalapino, Europhys. Lett. 30, 163 (1995); ibidem, cond-mat 9601047 (unpublished); D. J. Scalapino, Nature 377, 12 (1995).

[5] M. Fabrizio, Phys. Rev. B 48, 15838 (1993); D. V. Khveshchenko and T. M. Rice, Phys. Rev. B 50, 252 (1994); H. J. Schulz, Phys. Rev. B 53, R2959 (1996).

[6] L. Balents and M. P. A. Fisher, Phys. Rev. B 53, 12133 (1996).

[7] R. S. Eccleston et al., Phys. Rev. Lett. 73, 2626 (1994); K. Kojima et al., Phys. Rev. Lett. 74, 2812 (1995).

[8] M. Uehara et al., J. Phys. Soc. Japan 65, 2764 (1996).

[9] G. Timp, in Mesoscopic Phenomena in Solids, edited by B. L. Altshuler, P. A. Lee, and R. A. Webb (Elsevier, Amsterdam, 1990).

[10] We are using here the notation of the last of Refs. [5].

[11] E. Arrigoni, Phys. Lett. A 215, 91 (1996).

[12] Details will be given elsewhere: E. Arrigoni and B. Brendel and W. Hanke (unpublished).

[13] E. Orignac and T. Giamarchi, Phys. Rev. B 53, R10453 (1996); ibidem, cond-mat 9704064.

[14] We restrict our analysis to values of the doping corresponding to the inner C1S0 phase of Ref. [6].

[15] T. Kimura, K. Kuroki, and H. Aoki, Phys. Rev. B 51, 13860 (1995).

[16] Here and in the following we deal, for simplicity, with the potentials $v_{0}$ and $v_{2}$ only, since including the other terms (interband $v_{0, \pi}$ and other terms as relevant as $v_{2}$ ) does not change the form of $\rho(T)$ in Fig. 2.

[17] In particular, we estimate the contribution from (ii) by stopping the RG flow at the point $l=l^{*}$ where the strong-coupling regime is reached. To estimate the $U$ dependence of $\delta K_{\rho,+}$ we further add the bare values of the coupling [12]. This will be appropriate for $U \lesssim \pi v_{F}$.

[18] L. Balents and M. P. A. Fisher, Phys. Rev. B 55, R11973 (1997).

[19] T. Yanagisawa, Y. Shimoi, and K. Yamaji, Phys. Rev. B 52, R3860 (1995).

[20] All our results are valid down to $T \sim T_{\text {dis }}$.

[21] Unless otherwise specified, we consider $\Delta_{o} \sim \Delta_{s}$. Moreover, we assume both spin gaps to be equal.

[22] Assuming that the effective correlation exponents of the two spin and of the odd-charge modes are unity for $T \gg$ $\Delta_{s}, \Delta_{o}$ and neglecting terms of order $\left(U / t \times \Delta_{o} v_{F} / v_{F}\right)$.

[23] The ratio $c_{2} / c_{0}$ can be calculated similarly to Ref. [2]. For the parameters of Fig. 2 we obtain $c_{2} / c_{0} \approx 2.61$.

[24] Theoretical estimates place the $\mathrm{Sr}_{14-x} \mathrm{Ca}_{x} \mathrm{Cu}_{24} \mathrm{O}_{42-\delta}$ compound into the strong-coupling region (M. Imada, S. Maekawa, private communication).

[25] T. Osafune et al., Phys. Rev. Lett. 78, 1980 (1997).

[26] To evaluate $\rho(T)$ in the range $\Delta_{o} \leq T \leq \Delta_{s}$, we used the interpolation formula $v_{0}(T) \propto\left(T / E_{F}\right)^{\left(K_{\rho}+1\right) / 4-1}(T+$ $\left.\Delta_{s}\right)^{1 / 2} \exp -\Delta_{o} / T$, which gives the correct behavior for $v_{0}(T)$ (and thus for $\rho(T)$ ) in the regions $T \gg 2 \Delta_{s}$, $2 \Delta_{o} \ll T \ll 2 \Delta_{s}$, and $T \ll 2 \Delta_{o}$ (see text).

[27] N. Motoyama et al., Phys. Rev. B 55, R3386 (1997). 